\title{
Spectral Oscillation in Optical Frequency-Resolved Quantum-Beat Spectroscopy With a Few-Cycle Pulse Laser
}

\author{
Takayoshi Kobayashi and Zhuan Wang
}

\begin{abstract}
Impulsive stimulated resonant Raman scattering was induced by a 5.7 fs visible pulse from a non-collinear optical parametric amplifier. The pulse duration corresponded to 2.8 cycles of the central wavelength of the laser spectrum at $610 \mathrm{~nm}$. The induced spectral change was time-resolved with a 0.8 fs delay time step and the effective absorbance change $\Delta A(\lambda, t)$ was calculated from the normalized transmittance change $\Delta T(\lambda, t) / T$. The observed optical and vibrational $2-D$ spectrum was analyzed to separate the electronic and vibrational contributions to the transient difference absorption spectrum. The probe wavelength dependence of the vibrational amplitude was explained in terms of the coupling between the laser field, Stokes field, and the vibrational coordinates. Simultaneous measurement of both the time and wavelength dependence of the vibrational amplitudes clearly showed that the broadband spectra of the components corresponding to the laser and Stokes lights are oscillating in $\pi$ out of phase with the corresponding vibrational frequency. The probe delay time and probe wavelength dependence of the oscillation in the absorbance change induced by the femtosecond laser were fully analyzed. The results were explained by the imaginary and real part of the third-order susceptibility, respectively.
\end{abstract}

Index Terms-Few-cycle pulse, quantum beat, quantum beat spectroscopy, stimulated Raman, Stokes field, ultrashort pulse.

\section{INTRODUCTION}

Q UANTUM beat phenomena appears when two or more transitions interfere within smaller energy separation narrower than the pump energy spectrum. There are electronic quantum beats and vibrational quantum beats. Vibrational levels belonging to the same mode near a potential minimum exhibit almost the same separation, and so a clear wave packet

Manuscript received April 24, 2008; revised July 02, 2008. This work was supported in part by the Grant MOE ATU Program in NCTU, Japan. A part of this work was performed also under the joint research project of the Laser Engineering, Osaka University, under Contract Subject B1-27.

T. Kobayashi is with the Department of Applied Physics and Chemistry and Institute of Laser Science, University of Electro-Communications, Tokyo 182-8585, Japan, with the Japan Science and Technology Agency, Saitama 332-0012, Japan, with the Institute of Laser Engineering, Osaka University, Osaka 565-0871, Japan, and also with the Department of Electrophysics, National Chiao Tung University, Hsinchu 30010, Taiwan (e-mail: kobayashi@ils.uec.ac.jp).

Z. Wang is with the Department of Applied Physics and Chemistry and Institute of Laser Science, University of Electro-Communications, Tokyo 182-8585, Japan, and also with the Japan Science and Technology Agency, Saitama 3320012, Japan (e-mail: wang@ils.uec.ac.jp).

Color versions of one or more of the figures in this paper are available online at http://ieeexplore.ieee.org.

Digital Object Identifier 10.1109/JQE.2008.2003492 (a)

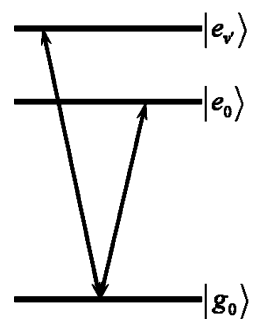

(b)

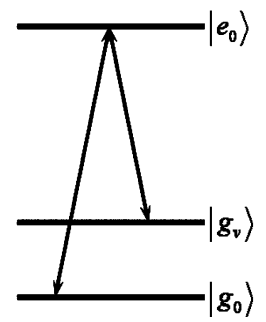

(c)

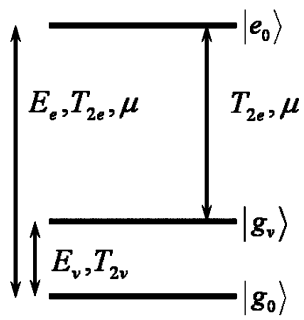

Fig. 1. Three-level systems. (a) $V$-type. (b) $\Lambda$-type. (c) Three-level model and parameters. The parameters are as follows. $E_{e}$, absorption energy of 0-0 transition; $E_{v}$, vibrational energy; $T_{2 e}$, electronic transverse dephasing time between the ground state and the excited state; $T_{2 v}$, vibrational transverse dephasing time between the vibrational states in the ground state; and $\mu$, transition dipole moment between the ground state and the excited state. In (a) $\left|g_{0}\right\rangle,\left|e_{0}\right\rangle$, and $\left|e_{v}\right\rangle$ are the ground electronic state, the lowest electronic excited state, and vibronic state of electronic energy of $E_{e}$ and vibrational energy of $E_{v}$, with vibrational quantum number $v^{\prime}$. In (b) $\left|g_{0}\right\rangle$ and $\left|e_{0}\right\rangle$ are the same in (a), $\left|g_{v}\right\rangle$ is the vibrational level of quantum number $v$ in the ground electronic state.

with a well-defined quantum beat can be generated unlike electronic quantum beat frequencies where nearly equal spacing of adjacent states cannot usually be expected.

Quantum-beat spectroscopy was first used to determine the spectral structure of nearby electronic states in atomic systems with a small gap that may be difficult to resolve in the frequency domain [1], [2]. The transient coherence between the sublevels of an excited atomic state induced by a short resonant light pulse appears in quantum beats in spontaneously emitted fluorescence light. This phenomenon is typically expected to be observed for a coupled three-level system as shown in Fig. 1(a) ( $V$-type); under normal experimental conditions it does not occur in the three-level system shown in Fig. 1(b) ( $\Lambda$-type). It is shown that coherence between the non-degenerate sublevels of an atomic ground state is efficiently induced with a short resonant light pulse [1], [2].

An even broader application of the vibration of molecular systems [3]-[14] has become feasible by the recent development of ultrashort pulses with a duration of less than a few tens of femtosecond, which is less than the typical oscillation periods of molecular vibration modes. Even incoherent pulses with longer duration but shorter coherence time than the vibration period were used to observe real-time molecular vibration quantum beats [15], [16]. This "incoherent" method was subsequently also applied to the electronic quantum beat caused by the small energy spacing in atomic systems [13], [14]. The 
molecular motion induced by an intense, ultrashort light pulse gives rise to an oscillatory modulation $\delta \Delta T$ (or quantum beat) in the transient transmittance change $\Delta T$. This $\Delta T$ signal can be caused by the induced absorption from excited states and/or reaction intermediates, stimulated emission from the excited states, and bleaching resulting from ground-state depletion. All of the above can be measured with the pump-probe technique.

Vibrational quantum-beat spectroscopy is sometimes called "real-time vibrational" spectroscopy or simply "real-time" spectroscopy. These names arise from the fact that the spectroscopy signal corresponds to the real-time oscillatory change in electronic transition intensity induced by molecular vibration. In this type of experiment, beats in organic molecules in solution with a frequency extending from several tens to a few thousand wavenumbers have been measured [3], [4], [7], [8], [17]-[19].

The wave packet can be generated either in the excited state via the simultaneous coherent excitation of vibronic polarizations of several (at least two) vibrational levels or in the ground state via "impulsive" stimulated Raman scattering (ISRS) [20]-[31]. These interactions, which are represented in terms of $V$-type and $\Lambda$-type, respectively, as shown in Fig. 1(a) and (b), are called a "split excited state" and a "split ground state," respectively.

A previously developed perturbative density-matrix formalism of quantum beats was extended to include three-level systems with ground-state splitting and with excited-state splitting, and four-level system with both [17], [18]. It was shown that we should be able to observe quantum beats in a transmission-correlation experiment when the optical pulses are resonant or non-resonant with the excited state. The beats may have a magnitude comparable to those from a split excited state. In addition, they are insensitive to the amount of homogeneous or inhomogeneous broadening of the optical transitions [17], [18]. This method can be used to determine the electronic dephasing time as proposed by Walmsley et al. [18].

It has been shown that there is no laser intensity threshold in the ISRS when a sufficiently short laser pulse is introduced to transmit through various types of matter. The ISRS excitation of coherent optical phonons [5], [6], molecular vibrations [24], [25], and other excitations (including rotational, electronic, and spin) may play important roles in femtosecond pulse interactions with molecules, crystals, glasses (including optical fibers), semiconductors, and metals [5], [6], [9].

In an electronic non-resonant condition the scheme of the interaction is simple [17]-[19]. Part of the probe beam spectrum is alternately Stokes shifted and anti-Stokes shifted by its interaction with the nuclear vibrational motion induced by the pump pulse. Therefore, the energy on the Stokes side of the peak of the probe spectrum is expected to be modulated out of phase with the energy on the anti-Stokes side. However, with electronic resonance the probe wavelength dependence of phase became complicated [17]-[19]. This is also a case of electronic resonance in the quantum beats. One important difference between the resonant and non-resonant cases is that the beats occur in the absorption of the probe pulse rather than the spectral redistribution of the probe pulse energy. In both cases, however, the beats arise from a coherent interaction of the probe with an impulsively excited phonon or coherent wave packet. There have been many general studies on more complicated systems composed of multi-vibrational levels of multi-vibrational modes. By using a Fourier transform (FT) of the vibrational real-time spectrum, the dynamics of the vibrational modes including the absolute vibrational phase and the phase relaxation time can be studied [26]-[28].

Conventional time-resolved Raman scattering spectroscopy and infrared (IR) absorption spectroscopy have two drawbacks. They can be used to study time-resolved molecular structural changes but in some cases the assignment of molecular vibration to the electronic state differs. This is because the dynamics of the electronic state is generally studied by another (electronic) type of time-resolved spectroscopy such as conventional pumpprobe absorption spectroscopy. The other disadvantage of the conventional methods is that they cannot provide the vibrational information.

With vibrational real-time spectroscopy, both of these difficulties can be overcome simultaneously. Since (1) the above information can be obtained with this method, the problem is completely solved, and (2) the vibrational phase can be determined by detecting the vibrational amplitude determined by the electronic transition probability change.

A simple physical model of the beats is one where the first pulse excites nuclear motion in a molecule. For this to take place, the energy spectrum of the pulse must overlap with at least two of the vibrational eigenlevels to introduce a coherent superposition of the levels. The second pulse probes the induced nuclear motion. For both the split excited and split ground states shown in Fig. 1, there is a change in the absorbance of the molecule, which depends on its delay from the pump pulse, because the electronic polarizability of the molecule varies with time due to the nuclear motion. This is immediately followed by an electronic distribution change.

Real-time spectroscopy can be extended even further to spectrally resolved real-time molecular vibration spectroscopy. Both the vibrational phase spectrum and amplitude spectrum were utilized for the dynamic spectral change associated with the molecular vibration [26]. Real-time spectroscopy has applications in various research fields including nanotechnology and nanoscience. If we are to develop these research fields we must clarify the mechanisms of the dynamic processes taking place in such systems as molecules and quantum dots, which are the key elements of the nanosystems, by disclosing the elementary processes essential for nanoscopic phenomena. Of these dynamic processes, primary processes are the most important since they determine the direction of subsequent functional processes cascading into a nanoscopic size. These primary nanoscopic processes inevitably occur in femtosecond time regime since the time required for the nucleus in a molecule to exert motion is on this time scale. Therefore, femtosecond spectroscopy is expected to be particularly powerful for clarifying the nanoscopic 
process by direct observation of the primary process with femtosecond time resolution. In other words the quantum effect becomes dominant in processes in the femtosecond region.

A pump-probe experiment with a pump pulse that has a shorter duration than the oscillation period corresponding to the inverse of the energy separation of the neighboring states or levels can generate electronic or vibrational wave packets. Real-time vibrational amplitude has been observed with a vibrational wave packet generated by the quantum beat of the vibrational levels [15], [16], [21]-[23], [25]-[27].

With these schemes, impulsive excitations of wave packets by ultrashort pulses can offer important information on vibronic coupling, conformational changes, and photochemical reactions [5], [6], [27], [28]. In contrast to conventional stationary Raman scattering experiments, which are relevant to ground-state vibrations, information about excited-state vibrations can be obtained by real-time spectroscopy using ultrashort pulse lasers. An ultrashort laser pulse can in general coherently induce both ground-state and excited-state vibrations with longer periods than the pulse duration. The efficiency ratio of the two cases was discussed in terms of pulse duration and laser frequency with respect to vibrational period and electronic resonant frequency, respectively [32].

This quantum beat spectroscopy can be used as a very powerful method for the study of molecular vibration by a pump-probe method. There are varieties of applications as follows. 1) The method studies both electronic decay and vibrational dynamics together in the same configuration. The contribution of the vibrational mode to the most strongly coupled electronic state can be made. The vibrational amplitude profile can be used for providing information of the assignment of the vibrational mode. This offers a kind of Raman excitation profile information with much higher reliability and nearly continuous wavelength dependent information of the Raman signal. 2) It also provides the molecular structure in the excited state [33]-[35], chemical intermediate [36] states, or even transition state [37], [38]. This method is much more advantageous than time resolved vibrational spectroscopies, like time-resolved Raman scattering and infrared absorption. By the latter methods, there is no information can be obtained about vibrational phase but by the former it can be obtained.

There are two third-order nonlinear processes, A and B, as shown in Fig. 2 for the molecular system we are studying and laser we employ. The contribution of the imaginary part of the third-order nonlinear susceptibility to the nonlinear processes $\mathrm{A}$ and $\mathrm{B}$ are demonstrated in our previous paper [26]. In the present paper we demonstrate for the first time the spectrally resolved real-time measurement of impulsive resonant Raman scattering composed of both of the A and B processes with contribution both from the real and imaginary part of the third-order nonlinear susceptibility. The work provides us with information exclusively on the ground-state vibration dynamics, because of the condition of the laser spectrum and the absorption spectrum that we discuss later. The probe wavelength dependence of the vibrational amplitude can be explained using coupled equations

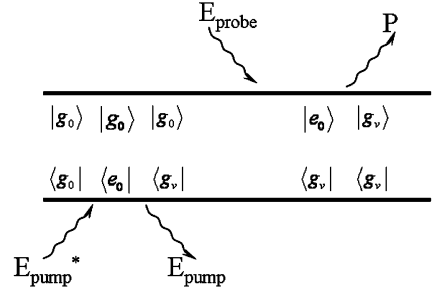

(a) NL process $A$

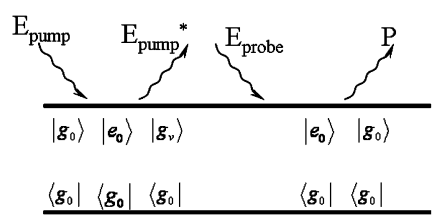

(b) NL process $B$

Fig. 2. Double-sided Feynman diagrams of the two nonlinear processes. $\left|g_{0}\right\rangle$, $\left|g_{v}\right\rangle$, and $\left|e_{0}\right\rangle$ are the same state as described in Fig. 1. In (a) (NL process A), the energy of the nonlinear polarization created by the final electronic coherence is lower than the absorption energy by the vibrational energy involved. In (b) (NL process $\mathrm{B}$ ), the energy of the nonlinear polarization is the same as the absorption energy.

for the laser field, Stokes field, and the vibrational coordinates [39]. The simultaneous measurement of both the time and wavelength dependencies of vibrational amplitudes clearly showed that the broadband spectra of the spectral components corresponding to the laser and Stokes lights are oscillating with an anti-phase relation. As a sample we used a quinoid thiophene derivative in tetrahydrofuran (THF) solution.

\section{EXPERIMENT}

The quinoid thiophene derivative, named as $\mathrm{Bx}-2$, used in the present study has dicyanomethylene groups at the terminal position and is a highly amphoteric redox molecule [40]. For the pump-probe experiment, the thiophene molecule was dissolved in tetrahydrofuran (THF), $0.02 \mathrm{wt} \%$, which give an absorbance peak of 2 at $480 \mathrm{~nm}$ in a 1-mm-thick quartz cell. The stationary absorption spectrum of Bx-2 shown in Fig. 3 was recorded with an absorption spectrometer (Shimadzu, model UV-3101PC).

The pulsed light source was a non-collinear optical parametric amplifier (NOPA) seeded with a white-light continuum [41]-[43]. The pump source of this NOPA system was a regenerative amplifier (Spectra Physics, Spitfire) with the following operating parameters: central wavelength, $805 \mathrm{~nm}$; pulse duration, $50 \mathrm{fs}$; repetition rate, $5 \mathrm{kHz}$; average output power, $740 \mathrm{~mW}$. The single filament continuum was generated by focusing a small intensity fraction of the amplifier output onto a 1-mm-thick sapphire glass plate. The NOPA output pulse was compressed with a pair of chirp mirrors and then with a prism pair, resulting in a nearly FT limited pulse duration of $5.7 \mathrm{fs}$. The pulse from the NOPA with a spectrum extending from 550 to $735 \mathrm{~nm}$ was split into two to obtain pump and probe pulses with energies of about 45 and $5 \mathrm{~nJ}$, respectively. The pump-probe experiment was performed with a delay time step of $0.8 \mathrm{fs}$ in a time range of $-200-1500 \mathrm{fs}$. The pump-probe signal was collected by a system combining a polychromator and multi-channel lock-in amplifier. The polychromator (300 grooves $/ \mathrm{mm}, 500 \mathrm{~nm}$ blazed) was utilized to disperse the probe 


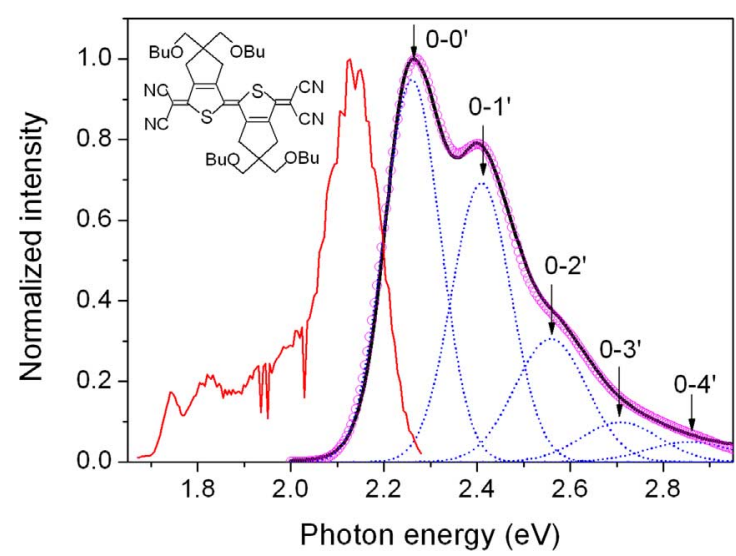

Fig. 3. Laser (thin solid line) and stationary absorption (thick solid line) spectrum. The dotted lines are the transition bands used in the Huang-Rhys fitting to the stationary absorption and the fitting results are plotted in open circle. $0-i^{\prime}$ $\left(i^{\prime}=0^{\prime}, 1^{\prime}, 2^{\prime}, 3^{\prime}\right.$, and $\left.4^{\prime}\right)$ is the transition from zeroth vibronic level in the ground state to the $i^{\prime}$ th level in the excited state. The inset shows the molecular structure of quinoid thiophene oligomer studied.

pulse and guide it to Avalanche photodetectors (APDs) by a 128-channel fiber bundle. The multichannel lock-in amplifier was designed for the purpose of detecting the signals simultaneously over the whole spectrum. Our system combining our sub-6 fs pulse laser light source with an extremely broad 4500 $\mathrm{cm}^{-1}$ bandwidth and our data collection system composed of a polychromator and a 128 channel lock-in amplifier is well suited to the study of broadband nonlinear processes A and B.

All experiments were performed at room temperature $(293 \pm$ $1 \mathrm{~K})$.

\section{THEORY}

The absorption and laser spectra are shown in Fig. 3 together with the molecular structure of the quinoid thiophene. The fractions of the laser power to the vibrational levels of quantum number $v^{\prime}$ in the electronic excited state in BX-2 were calculated to be $99 \%$ and $1 \%$ for $v^{\prime}=0^{\prime}$ and $1^{\prime}$, respectively. Therefore, we can consider that $v^{\prime}=1^{\prime}$ in the system is not excited by the 5.7 fs laser via a $\mathrm{V}$-type mechanism. Hence, the three-level model with several parameters shown in Fig. 1(c) can be safely used to analyze the $\Lambda$-type interaction. We utilized the theory of nonlinear ultrafast spectroscopy developed by Ducas et al. and Debeer et al. [11], [12], [14]. The two vibrational levels under consideration are in the electronic ground state $\left(\left|g_{0}\right\rangle\right.$ and $\left.\left|g_{v}\right\rangle\right)$ and one vibrational ground level with vibrational quantum number $v^{\prime}=0$ in an electronic excited state $\left|e_{0}\right\rangle$ as shown in Fig. 1(b) and (c). Interaction between the three-level system and the pump field $E_{\text {pump }}$ and probe field $E_{\text {probe }}$ can be depicted with double-sided Feynman diagrams [44] for the two third-order nonlinear processes (A and B) representing two different channels of coherent vibrational excitations in the ground state through the stimulated Raman process.

Other nonlinear processes leading to the excitation of the ground-state vibrations are not detected in a heterodyne pumpprobe experiment, because there is no spectral overlap between the probe pulse and the induced nonlinear polarizations. There are several other nonlinear processes, which contribute to nonlinear pump-probe signals such as coherent anti-Stokes Raman scattering (CARS), coherent Stokes Raman scattering (CSRS), bleaching of the ground state due to the depletion of the groundstate absorption, and induced absorption due to the generation of excited states and/or intermediate states. However, their dynamics is determined by the electronic longitudinal (population) relaxation time [44]-[46], which is usually much longer than the periods of typical molecular vibrations. This is because the electronic states are eigenstates, between which a radiationless transition takes place with the delay of vibronic coupling, or in other words via the distraction of the Born Oppenheimer approximation [47], [48]. Their contributions can be clearly separated in the following analysis of the vibrational dynamics. The examination of the diagrams in Fig. 2 leads to the following two important conclusions.

Firstly, the frequency difference between the nonlinear polarizations $\mathrm{A}$ and $\mathrm{B}$ corresponds to the energy difference $E_{v}$ between the two vibrational levels $\left|g_{v}\right\rangle$ and $\left|g_{0}\right\rangle$ shown in Fig. 1(c). This energy difference could be used to distinguish the nonlinear processes $\mathrm{A}$ and $\mathrm{B}$.

Secondly, the pump-probe signal at frequencies below the 0-0 transition can generally be modulated by both molecular vibration modes in the ground state and in the electronic excited state. Ground-state and excited-state dynamics can even coexist for frequencies above the 0-0 transition as a result of many nonlinear processes such as those in CSRS and CARS mentioned above. Therefore, in a conventional resonant pump-probe experiment, it is very difficult to find a spectral range within which the nonlinear signal will reflect excited-state dynamics exclusively.

In the present experiment as described above only the ground-state wave packet can be generated because of the conditions of the laser spectrum and the absorption spectrum of the sample. Therefore, the analysis has become much simpler. The pump-probe signal for nonlinear processes A and B were calculated from the nonlinear polarization with the Feynman diagrams shown in Fig. 2. The parameters describing the energy scheme in the figure are defined as follows. Fig. 1(c) shows the parameters and wave functions used in the present analysis. The energy, the transition dipole moment, and the transverse dephasing time between $\left|e_{0}\right\rangle$ and $\left|g_{0}\right\rangle$ are defined as $E_{e} \equiv \hbar \omega_{e}$, $\mu_{g e}$, and $T_{2 e}$, respectively. In the same way the energy between $\left|e_{0}\right\rangle$ and $\left|g_{v}\right\rangle$ is given by $E_{e}-E_{v} \equiv \hbar\left(\omega_{e}-\omega_{v}\right)$ and its transition dipole and dephasing time are give by $\mu_{g e}$ and $T_{2 e}$, respectively. The transverse vibration relaxation (vibrational dephasing) time between $\left|g_{0}\right\rangle$ and $\left|g_{v}\right\rangle$ is $T_{2 v}$. Since the pump and probe pulses are split from the same NOPA output by a beam splitter, they have the same pulse duration and the same center frequency. After the beam splitter, they propagate through different optical components and hence they may have different optical phases. The effect of the difference in the delay between the pump pulse and probe pulse caused by the group velocity dispersion induced by the beam splitter was compensated with great care with an appropriate compensator. It was 
designed and synthesized to have the same optical characteristics as the beam splitter. Therefore, even though the pump and probe pulses have different phases, their phase characteristic is the same. The phases of the pump and probe fields, $E_{\text {pump }}(t)$ and $E_{\text {probe }}(t)$ are defined as $\theta_{\text {pump }}$ and $\theta_{\text {probe }}$, respectively.

The vibrational real-time spectroscopy utilized in the present study is a kind of pump-probe experiment utilizing third-order nonlinearity. The typical ratio between the pump and probe pulse energy in our equipment was about 10 to 1 . Therefore, the contribution of the signal generated by the process whereby the pump and probe pulses interact with the sample once and two times is negligibly small. Hence, it can be safely assumed that the pump and probe pulses interact with the sample twice and once, respectively.

The effect of coherent coupling between the pump induced polarization and the probe field appears when the pump and probe pulses overlap. Another effect of the interaction between the probe induced polarization and the pump field appears when the probe pulse precedes [28], [49]-[54]. To avoid such effects we discuss only a case where the pump pulse precedes well ahead of the probe pulse without any overlap between them. In such a way only the pump-pump-probe sequence of the interaction between pulses and the sample is maintained using the rotating-wave approximation. The third-order nonlinear macroscopic polarizations of third-order nonlinear processes $A$ and $B$ are given by the following equation, which employs conventional perturbation theory using the Feynman diagram

$$
\begin{aligned}
P_{A}^{(3)}(t, \tau)= & \left(\frac{i}{\hbar}\right)^{3} \mu^{4} \int_{0}^{\infty} d t_{3} \int_{0}^{\infty} d t_{2} \int_{0}^{\infty} d t_{1} \\
& \times E_{\text {probe }}\left(t-t_{3}\right) E_{\text {pump }}\left(t-t_{3}-t_{2}+\tau\right) \\
& \times E_{\text {pump }}^{*}\left(t-t_{3}-t_{2}-t_{1}+\tau\right) \\
& \times \exp \left[-i\left\{\left(\omega_{e}-\omega_{v}-\omega_{0}\right) t_{3}-\omega_{v} t_{2}\right.\right. \\
& \left.\left.\quad-\left(\omega_{e}-\omega_{0}\right) t_{1}+\theta_{\text {probe }}\right\}\right] \\
& \times \exp \left(-\frac{t_{1}}{T_{2 e}}-\frac{t_{2}}{T_{2 v}}-\frac{t_{3}}{T_{2 e}}\right)
\end{aligned}
$$

and

$$
\begin{aligned}
P_{B}^{(3)}(t, \tau)= & \left(\frac{i}{\hbar}\right)^{3} \mu^{4} \int_{0}^{\infty} d t_{3} \int_{0}^{\infty} d t_{2} \int_{0}^{\infty} d t_{1} \\
& \times E_{\text {probe }}\left(t-t_{3}\right) E_{\text {pump }}^{*}\left(t-t_{3}-t_{2}+\tau\right) \\
& \times E_{\text {pump }}\left(t-t_{3}-t_{2}-t_{1}+\tau\right) .
\end{aligned}
$$

The duration of the pulse used in the experiment is about $6 \mathrm{fs}$, which is much shorter than the oscillation period of the vibrational modes of interest and the vibrational and electronic dephasing time. Therefore, the envelope functions of the pump and the probe electrical fields can be assumed to be $\delta$ functions. This makes the FT of (1) and (2) highly simplified. Because of the spectral distribution of the laser and the absorption spectrum of the sample, the nonlinear susceptibility satisfies both conditions of one-photon electronic resonance and two-photon difference frequency resonance. Therefore, the contributions of both the real and imaginary parts of the susceptibility make a substantial contribution to the modulation of the difference absorbance. The imaginary part is discussed below, followed by a discussion of the real part.

The induced incident pulse intensity change is proportional to the imaginary part of the nonlinear polarization multiplied by the conjugate of the incident electric field

$$
\Delta A_{X}(\omega, \tau) \propto-\operatorname{Im}\left[\tilde{P}_{X}^{(3)}(\omega, \tau) \tilde{E}_{\text {probe }}^{*}(\omega, \tau)\right] .
$$

Here $\tilde{P}_{X}^{(3)}(\omega, \tau)$ and $\tilde{E}_{\text {probe }}^{*}(\omega, \tau)$ are the FTs of $P_{X}^{(3)}(t, \tau)$ and $E_{\text {probe }}^{*}(t, \tau)$, respectively. Here suffix $X$ is either A or B corresponding to nonlinear process A or B. The change in the optical absorbance of the sample appearing as pump-probe signals for nonlinear processes A and B can be described in the following way when the transmittance change is sufficiently small

$$
\begin{aligned}
\Delta A_{A}(\omega, \tau) \propto & \frac{-\left(\omega_{e}-\omega_{v}\right) \operatorname{Im}\left[\tilde{E}_{\text {probe }}^{*}(\omega) \tilde{P}^{(3)}(\omega)\right]}{\left|\tilde{E}_{\text {probe }}(\omega)\right|^{2}} \\
\propto & \left(\omega_{e}-\omega_{v}\right) \exp \left(-\frac{\tau}{T_{2 v}}\right) \\
& \times \frac{-\Omega \sin \left(\omega_{v} \tau\right)+\gamma_{2} \cos \left(\omega_{v} \tau\right)}{\Omega^{2}+\gamma_{2}^{2}}
\end{aligned}
$$

and

$$
\Delta A_{B}(\omega, \tau) \propto \omega_{e} \exp \left(-\frac{\tau}{T_{2 v}}\right) \frac{\Omega^{\prime} \sin \left(\omega_{v} \tau\right)+\gamma_{2} \cos \left(\omega_{v} \tau\right)}{\Omega^{\prime 2}+\gamma_{2}^{2}} .
$$

where $\gamma_{2} \equiv 1 / T_{2 e}, \Omega=\omega-\omega_{e}+\omega_{v}$, and $\Omega^{\prime}=\omega-\omega_{e}$. The approximation $\Delta A_{X}(\omega, \tau) \propto\left(\Delta T_{X}(\omega, \tau) / T_{X}(\omega, \tau)\right)$ was used. Here $T_{X}(\omega, \tau)$ is the transmission of the probe pulse after the sample without the presence of the pump pulse, and $\Delta T_{X}(\omega, \tau)$ is the transmittance change with the presence of the pump pulse. The result was obtained because $\left(\Delta T_{X}(\omega, \tau) / T_{X}(\omega, \tau)\right)(X=$ $A, B)$ was sufficiently small in the present experiment.

The spectral width and the vibrational dephasing time in (4) and (5) are determined by the electronic dephasing time $T_{2 e}$ and the vibrational dephasing time $T_{2 v}$, respectively. Using this principle, the electronic dephasing time and the vibrational dephasing time can be separately determined. The advantage provided by the direct real-time measurement of the vibrational phase means that the results given by (4) and (5) also demonstrate the dependence of the periodic modulation phase on the probe frequency.

The molecular vibration information can be obtained by probing the modulated difference absorption $\Delta A(\omega, \tau)$ given by $\delta \Delta A(\omega, \tau) . \delta \Delta A(\omega, \tau)$ is the difference between the induced absorbance change at probe time $\tau$ and that at the equilibrium position of the molecular vibration after coherent molecular vibration has been excited. The modulation of difference absorbance $\Delta A_{X}\left(t, \omega ; \omega_{v}\right)$ with the angular frequency of the vibration $\omega_{v}$ and the delay time constant $\tau_{0}$ is given 
by the following equation as a function of the optical angular frequency $\omega$ and probe delay time $t$

$$
\begin{aligned}
\delta \Delta A_{X}\left(t, \omega ; \omega_{v}\right)=\delta \Delta A_{X}\left(\omega_{v}, \omega\right) & \exp \left(-\frac{t}{\tau_{0}\left(\omega_{v}, \omega\right)}\right) \\
& \times \cos \left[\omega_{v} \tau+\phi_{X}\left(\omega_{v}, \omega\right)\right] .
\end{aligned}
$$

Here suffix $X$ is either A or B, which corresponds to nonlinear process A or B. The two processes have different amplitudes $A_{X}$ and phases $\phi_{X}$. The amplitudes $A_{A}$ and $A_{B}$ are given by (4) and (5). The phases $\phi_{A}$ and $\phi_{B}$ are given by

$$
\begin{aligned}
& \phi_{A}\left(\omega_{v}, \omega\right)=\arctan \left(\frac{\omega-\left(\omega_{e}-\omega_{v}\right)}{\gamma_{2}}\right) \\
& \phi_{B}\left(\omega_{v}, \omega\right)=\arctan \left(\frac{-\left(\omega-\omega_{e}\right)}{\gamma_{2}}\right) .
\end{aligned}
$$

If the $\phi_{B}\left(\omega_{v}, \omega\right)$ spectrum is shifted downward by vibrational frequency

$$
\phi_{B}\left(\omega_{v}, \omega-\omega_{v}\right)=\arctan \left(\frac{-\left(\omega-\left(\omega_{e}-\omega_{v}\right)\right)}{\gamma_{2}}\right)
$$

then

$$
\phi_{A}\left(\omega_{v}, \omega\right)-\phi_{B}\left(\omega_{v}, \omega-\omega_{v}\right)=m \pi(m: \text { odd integer })
$$

Therefore, the signals resulting from nonlinear processes A and B have an anti-phase relation. The physical meaning of this phase relation is as follows. Depending on the phase of the molecular vibrational excited by the pump pulse at zero delay time, the spectrum in the $\omega_{e}$ region is amplified (deamplified) and that in the $\omega_{e}-\omega_{v}$ region is deamplified (amplified) alternatively. This is the energy exchange process between the probe pulse and the molecular vibration.

The difference in the phases reaches $\pi$ between the higherfrequency region $\left(|\Omega| \gg \gamma_{2}, \Omega>0\right)$ and the lower-frequency region $\left(|\Omega| \gg \gamma_{2}, \Omega<0\right)$. This is an appropriate criterion for identifying the ground-state dynamics and separation of the origin of the vibration in such a spectrally resolved real-time experiment [55]-[57].

Thus far we have discussed the effect of the imaginary part of the third order susceptibility on the absorbance change. The modulation, $\delta \Delta A\left(t, \omega ; \omega_{v}\right)$, induced by the molecular vibration of the difference absorbance, $\Delta A\left(t, \omega ; \omega_{v}\right)$, of the electronic transition can take place via absorbance (transmittance) change. This process involves either amplification or deamplification of the probe pulse by the coherent molecular vibration depending on the phase of the vibration as mentioned above. Since the time-resolved difference absorption spectrum given by (3) is contributed by the imaginary part of the product of $\tilde{P}_{X}^{(3)}(\omega, \tau)$ and $\tilde{E}_{\text {probe }}^{*}(\omega, \tau)$, it is dominated by the absorbed spectrum of the probe light by the sample.

On the other hand the real part of the third-order susceptibility can also contribute to the modulation via a probe pulse spectral change induced by the cross phase modulation mechanism. This mechanism results from the refractive index change

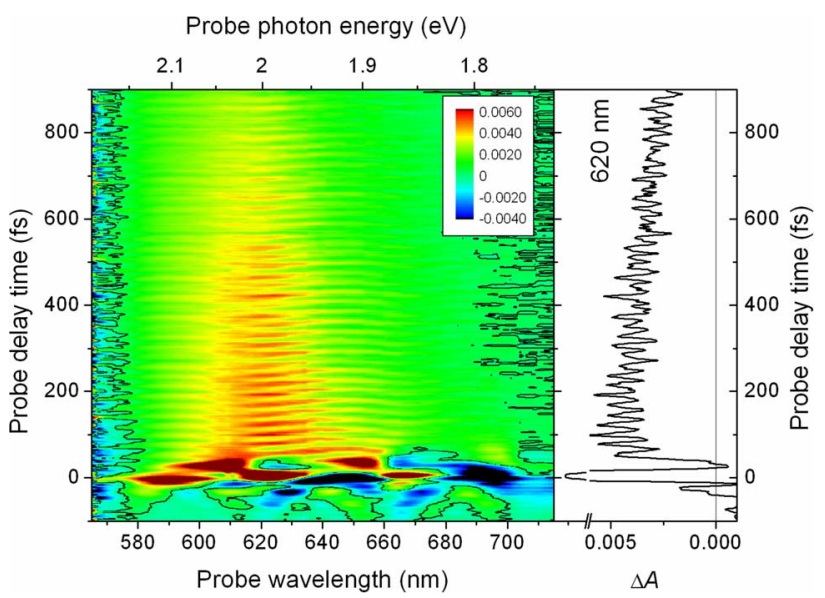

Fig. 4. 2-D real-time absorbance change $\Delta A(\lambda, t)$. The legend indicate $\triangle A(\lambda, t)$ the intensity. One example of the time dependent absorbance change at the probe wavelength of $620 \mathrm{~nm}$ was plotted in the right column.

caused by the deformation of the molecular configuration during molecular vibration. The effect of the real part of the third order susceptibility on the absorbance change is expected to be similar to that of the imaginary part because of the Kramers-Kronig relation.

\section{EXPERIMENTAL RESULTS AND DISCUSSION}

Fig. 3 shows the laser spectrum and the absorption spectrum of the sample quinoid-type thiophene oligomer. The absorption band was separated into four vibrational progression subbands. These sub bands correspond to the transition from the vibration level $v=0$ in the ground state to the excited state vibration level with quantum numbers $v^{\prime}=0^{\prime}, 1^{\prime}, 2^{\prime}, 3^{\prime}$, and $4^{\prime}$, as shown in Fig. 3. From the spectral overlap, $10.2 \%$ and $0.07 \%$ of the NOPA output energy was calculated to be absorbed by the $0-0^{\prime}$ and $0-1^{\prime}$ transitions, respectively. Therefore, $99 \%$ of the absorbed pump energy was utilized to excite the vibrational level with a quantum number of $0^{\prime}$ in the excited state and only $1 \%$ was utilized to excite the vibrational level with a quantum number of $1^{\prime}$. This means that there is no excited-state wave-packet formation through the V-type interaction [Fig. 1(a))]in this system.

Fig. 4 shows the probe delay time $(t)$ and probe frequency $(\omega)$ dependences of the absorbance change $(\Delta A(t, \omega))$. One example of the time dependent absorbance change at a probe wavelength of $620 \mathrm{~nm}$ is plotted in the right column. The photon induced absorbance change $\Delta A$ is positive in a spectral range of 574 to $700 \mathrm{~nm}(2.16-1.77 \mathrm{eV})$ and negative beyond this spectral range. The positive photo induced absorbance is due to the induced absorption from the excited state and the negative signal is caused by the absorption bleaching owing to the ground state depletion. Fig. 4 clearly shows the periodic modulation of the absorbance change as a function of probe delay time. This modulation is caused by the transition probability change induced by molecular vibration. The FT of this periodical change will provide information corresponding to the vibrational amplitude and phase. 


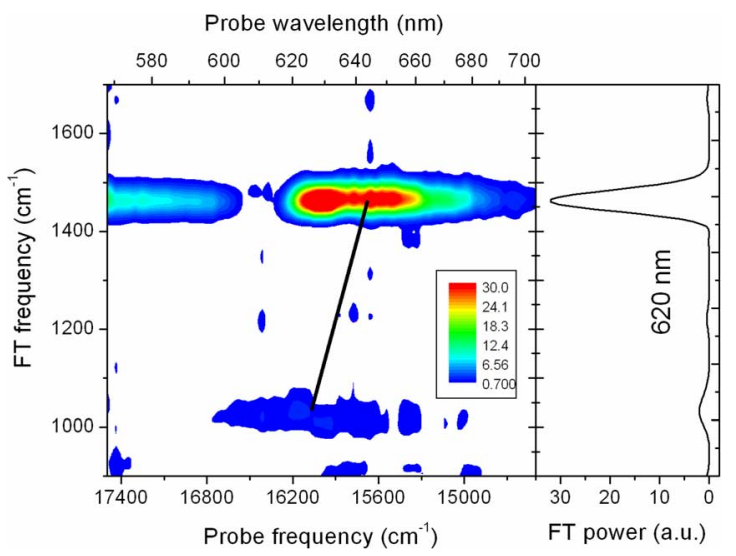

Fig. 5. 2-D FT power spectra. Solid line combines the center of masses of the FT power spectrum for modes of 1037 and $1460 \mathrm{~cm}^{1}$. The center of mass for mode $1037 \mathrm{~cm}^{1}$ was calculated after Lorentzian fitting to the FT power spectrum. One example of FT power spectrum at the probe wavelength of $620 \mathrm{~nm}$ was plotted in the right column.

The FT was performed in the following way. The real-time traces were smoothed by adjacent averaging to obtain slow electronic relaxation. When this slow electronic relaxation was subtracted from the real-time traces, the vibrational dynamics remained, and these were used to calculate the FT. In the present study, we used a 200 fs time range, which gave a cutoff frequency of $167 \mathrm{~cm}^{-1}$. This is because the cutoff frequency was determined by the time range of the data point used in the adjacent averaging. The FT power spectrum is displayed 2-Dly against the probe photon energy and FT frequency in Fig. 5. There are two prominent vibrational modes with frequencies at $v_{1}=1037 \mathrm{~cm}^{-1}$ and $v_{2}=1460 \mathrm{~cm}^{-1}$. They can be attributed to the $\mathrm{C}-\mathrm{C}$ and $C=C$ stretching modes. These modes may be expressed as being mixed with other local modes.

We analyzed the effect of the imaginary part of the susceptibility in a similar way to that in our previous paper [26]. Broad signals were found around 570 and $638 \mathrm{~nm}$, and $622 \mathrm{~nm}$ for the 1460 and $1037 \mathrm{~cm}^{-1}$ modes, respectively, which clearly shows the probe wavelength dependence indicated by (4) even though there is no clear signal for the $1037 \mathrm{~cm}^{-1}$ mode on the high photon energy side. The peaks around $638 \mathrm{~nm}$ and $622 \mathrm{~nm}$ correspond to $\omega_{e}-\omega_{v 1}$ and $\omega_{e}-\omega_{v 2}$ in nonlinear process $\mathrm{A}$. The peak of $570 \mathrm{~nm}$ for the $1460 \mathrm{~cm}^{-1}$ mode corresponds to $\omega_{e}$ in nonlinear process B. We calculated the slope of the solid line shown in Fig. 5 connecting the center of mass of the FT powers of the mode with frequencies of 1460 and $1037 \mathrm{~cm}^{-1}$ (peaks at $638 \mathrm{~nm}$ and $622 \mathrm{~nm}$ ). The centers of mass of the FT amplitude spectra determined by $\Omega$ in (4) are related to the vibrational frequency $\omega_{v}$. Therefore, the slope $K=\left(\omega_{v 1}-\omega_{v 2} / \Omega_{1}-\Omega_{2}\right)=$ $\left(\omega_{v 1}-\omega_{v 2} / \omega_{v 1}-\omega_{v 2}\right)=1$ was expected. The experimental slope was calculated to be $1.05 \pm 0.05$, which is sufficiently close to the expected value, thus providing evidence of the signal being due to nonlinear process A.

The FT amplitude spectra (FT-AS) for the 1037 and $1460 \mathrm{~cm}^{-1}$ modes are extracted from Fig. 5 and plotted as a function of probe photon energy in Fig. 6(a) and (b), respectively. In the figures, the laser spectrum (LS), the absorbed

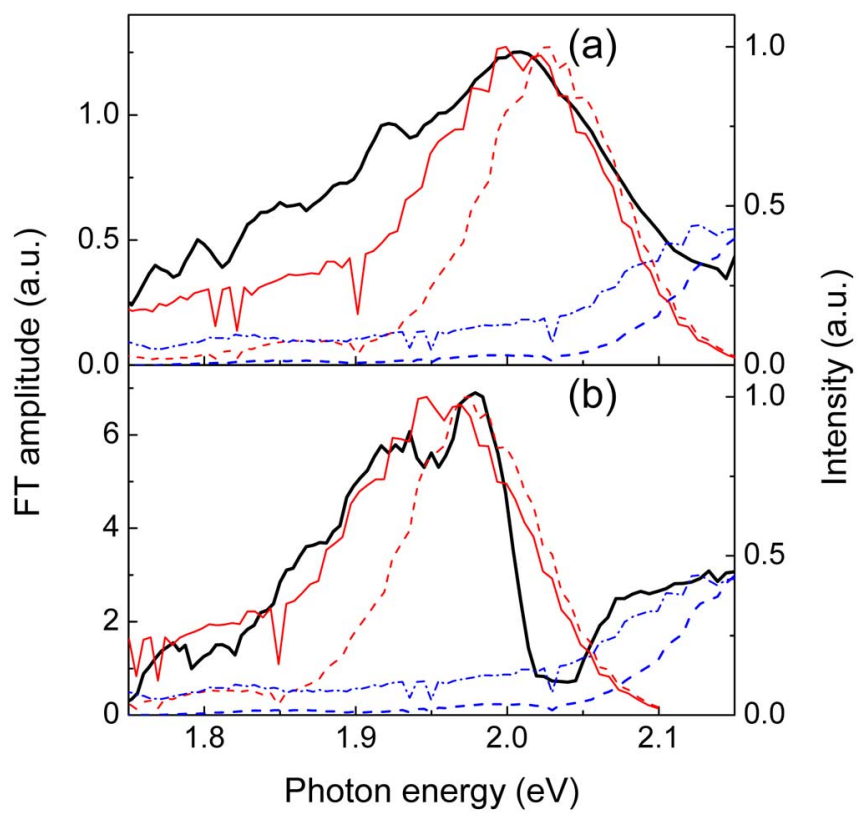

Fig. 6. (a)-(b) For vibration modes of 1037 and $1460 \mathrm{~cm}^{-1}$, respectively. The line information are: FT amplitude spectra (thick solid line), laser spectra (dash dotted line), and absorbed spectra (blue dotted line). And the shifted laser and absorbed spectra by the amount of the laser spectrum is plotted in thin solid and thin dashed lines, respectively, for modes of 1037 and $1460 \mathrm{~cm}^{-1}$.

probe spectrum (APS), and the LS and APS downshifted to the lower photon energy side by 1037 and $1460 \mathrm{~cm}^{-1}(0.128$ and $0.181 \mathrm{eV}$ ) are also shown. The absorbed probe spectrum is calculated from the sample absorption spectrum and the laser spectrum. Fig. 6 shows that the FT-AS of a mode with a frequency of $1460 \mathrm{~cm}^{-1}$ fitted relatively well with the LS and shifted LS on the higher and lower photon energy sides (nonlinear processes B and A), respectively. For the $1037 \mathrm{~cm}^{-1}$ mode, the FT-AS also fitted well with the shifted LS on the lower photon energy side (nonlinear process A). This result is different from that in our previous paper [26] where the shifted APS fitted well with the observed FT-AS of the relevant mode in a conjugated polymer, polydiacetylene. This indicates that a consideration of only the imaginary part of the third-order susceptibility is insufficient in the present study. And the real part must also be considered.

As mentioned above, the real part of the susceptibility can also contribute to $\delta \Delta A_{X}(\omega, \tau)$ both the $X=A$ and B nonlinear processes through cross-phase modulation. The crossphase modulation is induced by the modulated refractive index from the electronic nonlinearity change as a result of the change molecular structure caused by the molecular vibration. Thus, the effect of the real part of the cross phase modulation is spectral shift in difference absorbance spectra, which is given by the first derivative of the laser spectrum. The $1460 \mathrm{~cm}^{-1}$ mode was used to analyze the effect of the real part of the susceptibility. The effect of the imaginary part was subtracted by taking the difference between the FT-AS and the downshifted APS in the spectral range below $2.03 \mathrm{eV}$ (nonlinear process A). The difference 


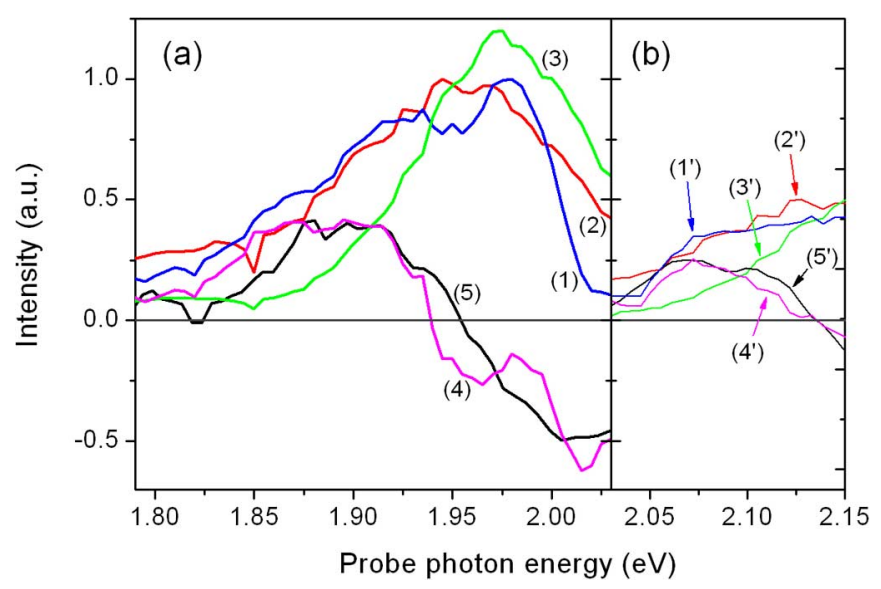

Fig. 7. (a) FT amplitude spectrum (line 1,blue), shifted laser spectrum (line 2, red), shifted absorbed spectrum (line 3 , green), the difference between curve (2) and (3) (line 4, pink), and the first order derivative of curve (2) (line 5, black). (b) FT amplitude spectrum (line $1^{\prime}$, blue), laser spectrum (line $2^{\prime}$, red), absorbed spectrum (line $3^{\prime}$, green), the difference between curve $\left(2^{\prime}\right)$ and $\left(3^{\prime}\right)$ (line $4^{\prime}$, pink), and the first order derivative of curve $\left(2^{\prime}\right)$ (line $5^{\prime}$, black).

between FT-AS and the APS was also calculated in the spectral range above $2.03 \mathrm{eV}$ (nonlinear process $\mathrm{B}$ ). The difference spectrum is temporarily called the "residual" spectrum, and the results are shown in Fig. 7(a) and (b). In order to evaluate this effect, we calculated the first derivative of the LS and the shifted LS as shown in Fig. 7(a) and (b). The agreement of this spectrum with the "residual" spectrum means that the Fourier amplitude spectrum of the $1460 \mathrm{~cm}^{-1}$ mode is explained in terms of the APS being downshifted by $1460 \mathrm{~cm}^{-1}$ and the first derivative of the LS being downshifted by the same amount in the nonlinear process A. A similar conclusion could be also made for the nonlinear process B. This result indicates that both the real and imaginary parts of the third-order nonlinear susceptibility have been observed in two nonlinear processes A and B in the present study using 2-Dly probed spectroscopy.

The modulation phases remain to be discussed. In Figs. 5 and 6(b) there is a dip around $2.03 \mathrm{eV}$ in the FT spectrum. This is due to the phase relation between (7) and (8) of nonlinear processes $\mathrm{A}$ and $\mathrm{B}$, which have opposite signs. Therefore, in the overlap region of the spectra caused by nonlinear processes A and B, they cancel each other out resulting in a much weaker Fourier power than expected for the sum of the contributions of the laser spectrum. As shown in Fig. 8, the experimentally observed vibrational phase spectrum is relatively complicated. As discussed by Walmsley et al. [17], when electronic resonance is involved, the probe wavelength dependence of the vibrational phase has complicated features. The present experimental data cannot be explained simply in terms of (7) and (8). Therefore, the difference between $\phi_{A}\left(\omega_{v}, \omega\right)$ and $\phi_{B}\left(\omega_{v}, \omega\right)$ was very difficult to identify directly. However, with the help of (10) the phase relation between nonlinear processes $\mathrm{A}$ and $\mathrm{B}$ was clearly shown.

From (7) and (8), nonlinear process A has a central frequency at $\left(\omega_{e}-\omega_{v}\right)$ and nonlinear process B has a central frequency at $\omega_{e}$. Therefore, in the present study, we considered that the

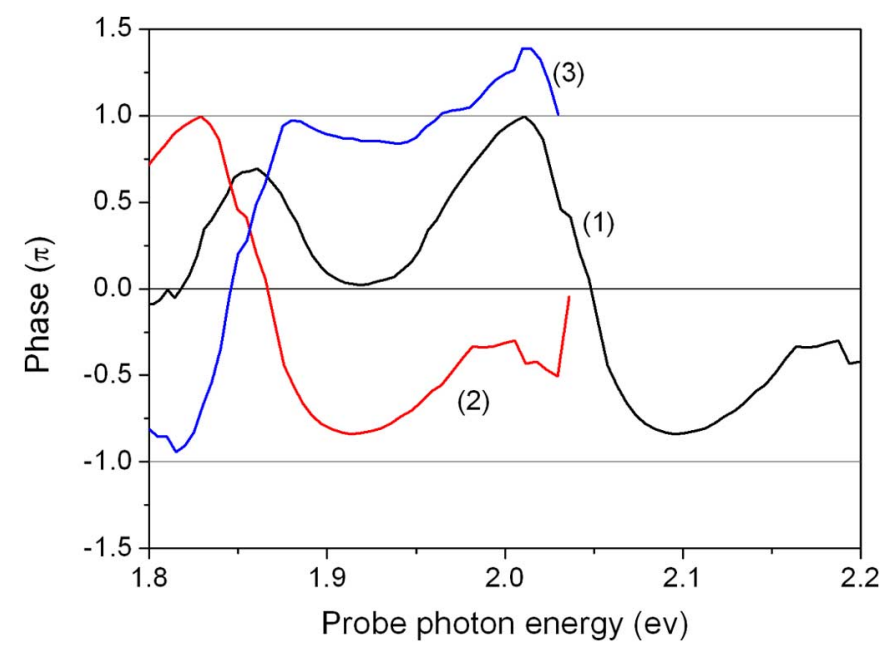

Fig. 8. Phase spectra for mode $1460 \mathrm{~cm}^{-1}$ [line (1)], the shifted phase spectra to the low photon energy side by the amount of vibration frequency $1460 \mathrm{~cm}^{-1}$ [line (2)], anddifference spectra calculated from (1) and (2) [line (3)].

phase spectrum in the spectral range $1.8 \sim 2.0 \mathrm{eV}$ represents the phase spectrum of nonlinear process $\mathrm{A} \phi_{A}\left(\omega_{v}, \omega\right)$. And the phase spectrum in the spectral range $2.0 \sim 2.2 \mathrm{eV}$ was considered to represent the phase spectrum of nonlinear process $B$ $\phi_{B}\left(\omega_{v}, \omega\right)$. The phase term $\phi_{B}\left(\omega_{v}, \omega-\omega_{v}\right)$ could be obtained by downshifting the phase spectrum $\phi_{B}\left(\omega_{v}, \omega\right)$ by $1460 \mathrm{~cm}^{-1}$. Fig. 8 clearly shows that the difference between $\phi_{A}\left(\omega_{v}, \omega\right)$ and $\phi_{B}\left(\omega_{v}, \omega-\omega_{v}\right)$ in the $1.8 \sim 2.0 \mathrm{eV}$ spectral range is $\pm \pi$, which is consistent with (10).

The results of the FT amplitude and FT phase analysis both reveal the coexistence of nonlinear processes of A and B. The real part of the third order nonlinear susceptibility to these two nonlinear processes mediated by molecular vibration was observed for the first time. Both amplitude and phase are well explained by the theoretical analysis.

\section{CONCLUSION}

A femtosecond pump-probe experiment was performed on a quinoid thiophene oligomer, in which the impulsive stimulated resonant Raman scattering signal was time-resolved with a 0.8 fs delay time step. The absorbance change of the sample after short pulse excitation was obtained with a multi-channel detector. The theoretical model was described with nonlinear processes A and B performed by broadband impulsive stimulated Raman scattering. A vibrational phase analysis showed that the broadband vibrational phase spectra of the components corresponding to laser and Stokes lights were oscillating in $\pi$ out of phase with the corresponding vibrational frequency, which indicates the coexistence of nonlinear processes A and B. A vibrational amplitude analysis showed that both the imaginary and real parts of the third-order susceptibility contributed to the modulated difference absorbance. We could thus for the first time observe the imaginary part contribution to the the nonlinear processes in the 2-D oscillating spectrum of the transmitted probe light. 


\section{ACKNOWLEDGMENT}

The authors are grateful to T. Otsubo, Prof. Dr. Emeritus of Hiroshima University for providing us with the QT2 molecule and Prof. E. Tokunaga for his help with the Raman scattering measurements.

\section{REFERENCES}

[1] J. Mlynek and W. Lange, "A simple method of observing coherent ground state transients," Opt. Comm., vol. 30, no. 3, pp. 337-340, 1979.

[2] H. Harde, H. Burggraf, J. Mlynek, and W. Lange, "Quantum beats in forward scattering: Subnanosecond studies with a mode-locked dye laser," Opt. Lett., vol. 6, no. 6, pp. 290-290, 1981.

[3] A. Laubereau, G. Wochner, and W. Kaiser, "Ultrafast coherent excitation and probing of molecular vibrations with isotopic substructure," Phys. Rev. A, vol. 13, no. 6, pp. 2212-2225, 1976.

[4] W. Zinth, "Transient coherent Raman scattering in the time and frequency domain," Opt. Comm., vol. 34, no. 3, pp. 479-482, 1980.

[5] K. A. Nelson, R. Casalegno, R. J. D. Miller, and M. D. Fayer, "Laser-induced excited state and ultrasonic wave gratings: Amplitude and phase grating contributions to diffraction," J. Chem. Phys., vol. 77, pp. 1144-1152, 1982.

[6] Y. X. Yan, E. B. Gamble, Jr, and K. A. Nelson, "Impulsive stimulated scattering: General importance in femtosecond laser pulse interactions with matter, and spectroscopic applications," J. Chem. Phys., vol. 83, pp. 5391-5399, 1985.

[7] M. J. Rosker, F. W. Wise, and C. L. Tang, "Femtosecond relaxation dynamics of large molecules," Phys. Rev. Lett., vol. 57, pp. 321-324, 1986.

[8] F. W. Wise, M. J. Rosker, and C. L. Tang, "Oscillatory femtosecond relaxation of photoexcited organic molecules," J. Chem. Phys., vol. 86, pp. 2827-2832, 1987.

[9] S. Ruhman, A. G. Joly, and K. A. Nelson, "Time-resolved observations of coherent molecular vibrational motion and the general occurrence of impulsive stimulated scattering," J. Chem. Phys., vol. 86, pp. 6563-6565, 1987.

[10] J. Mlynek, W. Lange, H. Harde, and H. Burggraf, "High-resolution coherence spectroscopy using pulse trains," Phys. Rev. A, vol. 24, pp. 1099-1102, 1981.

[11] T. W. Ducas, M. G. Littman, and M. L. Zimmermann, "Observation of oscillations in resonance absorption from a coherent superposition of atomic states," Phys. Rev. Lett., vol. 35, pp. 1752-1754, 1975.

[12] D. Debeer, L. G. Van Wagenen, R. Beach, and S. R. Hartmann, "Ultrafast modulation spectroscopy," Phys. Rev. Lett., vol. 56, pp. $1128-1131,1986$

[13] J. E. Golub and T. W. Mossberg, "Ultrahigh-frequency interference beats in transient, incoherent-light four-wave mixing," Opt. Lett., vol. 11, pp. 431-434, 1987.

[14] D. Debeer, E. Usadi, and S. R. Hartmann, “Attosecond beats in sodium vapor," Phys. Rev. Lett., vol. 60, pp. 1262-1265, 1988.

[15] T. Hattori, A. Terasaki, and T. Kobayashi, "Coherent Stokes Raman scattering with incoherent light for vibrational-dephasing-time measurement," Phys. Rev. A, vol. 35, pp. 715-724, 1987.

[16] K. Kurokawa, T. Hattori, and T. Kobayashi, "Subpicosecond molecular dynamics studied by degenerate four-wave mixing with incoherent light," Phys. Rev. A, vol. 36, pp. 1298-1304, 1987.

[17] I. A. Walmsley, M. Mitsunaga, and C. L. Tang, "Theory of quantum beats in optical transmission-correlation and pump-probe experiments for a general Raman configuration," Phys. Rev. A, vol. 38, pp. 4681-4689, 1988.

[18] I. A. Walmsley and C. L. Tang, "The determination of electronic dephasing rates in time-resolved quantum-beat spectroscopy," J. Chem. Phys., vol. 92, pp. 1568-1574, 1990.

[19] M. Mitsunaga and C. L. Tang, "Theory of quantum beats in optical transmission-correlation and pump-probe measurements," Phys. Rev. A, vol. 35, pp. 1720-1728, 1987.

[20] J. Ha, M. Maris, W. Risen, J. Tauc, C. Thomson, and Z. Vardeny, "Observation of molecular vibrations in real time," Phys. Rev. Lett., vol. 57, pp. 3302-3302, 1986.
[21] K. A. Nelson and L. WIllianms, "Femtosecond time-resolved observation of coherent molecular vibrational motion," Phys. Rev. Lett., vol. 58, pp. 745-745, 1987.

[22] S. Ruhman, A. G. Joly, and K. A. Nelson, "Coherent molecular vibrational motion observed in the time domain through impulsive stimulated Raman scattering," IEEE J. Quant. Electron., vol. 24, pp. 460-469, 1988.

[23] S. Ruhman, A. G. Joly, B. Kohler, L. R. Williams, and K. A. Nelson, "Intramolecular and intermolecular dynamics in molecular liquids through femtosecond time-resolved impulsive stimulated scattering," Rev. Phys. Appl., vol. 22, pp. 1717-1734, 1987.

[24] J. Chesnoy and A. Mokhtari, "Oscillations ultrarapides de l'absorption et de la biréfringence photoinduites observées par une technique de modulation temporelle," Rev. Phys. Appl., vol. 22, pp. 1743-1748, 1987.

[25] A. Mokhtari and J. Chesnoy, "Resonant impulsive stimulated Raman scattering," Europhys. Lett., vol. 5, pp. 523-528, 1988.

[26] N. Ishii, E. Tokunaga, S. Adachi, T. Kimura, H. Matsuda, and T. Kobayashi, "Optical frequency- and vibrational time-resolved 2-D spectroscopy by real-time impulsive resonant coherent Raman scattering in polydiacetylene," Phys. Rev. A, vol. 70, pp. 023811-023811, 2004.

[27] T. Kobayashi, A. Shirakawa, H. Matsuzawa, and H. Nakanishi, "Realtime vibrational mode-coupling associated with ultrafast geometrical relaxation in polydiacetylene induced by sub-5-fs pulses," Chem. Phys. Lett., vol. 321, pp. 385-393, 2000.

[28] S. Adachi, V. M. Kobryanskii, and T. Kobayashi, "Excitation of a breather mode of bound soliton pairs in trans-polyacetylene by sub-five-femtosecond optical pulses," Phys. Rev. Lett., vol. 89, pp. 027401-027401, 2002

[29] G. Cerullo, G. Lanzani, M. Muccini, C. Taliani, and S. D. Silvestri, "Real-time vibronic coupling dynamics in a prototypical conjugated oligomer," Phys. Rev. Lett., vol. 83, pp. 231-234, 1999.

[30] G. Lanzani, G. Cerullo, S. Stagira, M. Zavelani-Rossi, and S. De Silvestri, "Photoexcitation of conjugated systems studied with sub-10 fs time resolution," Synth. Met., vol. 119, pp. 491-494, 2001.

[31] T. P. Dougherty, G. P. Wiederrecht, and K. A. Nelson, "Impulsive stimulated Raman scattering experiments in the polariton regime," J. Opt. Soc. Amer. B, vol. 9, pp. 2179-2189, 1992.

[32] C. J. Bardeen, Q. Wang, and C. V. Shank, "Selective excitation of vibrational wave packet motion using chirped pulses," Phys. Rev. Lett., vol. 75, pp. 3410-3413, 1995.

[33] H. Kano, T. Saito, A. Ueki, and T. Kobayashi, "First observation of dynamic intensity borrowing induced by coherent molecular vibrations in J-aggregates revealed by sub-5-fs spectroscopy," Int. J. Mod. Phys. $B$, vol. 15 , pp. $3817-3820,2001$.

[34] H. Kano, T. Saito, and T. Kobayashi, "Dynamic intensity borrowing in porphyrin J-aggregates revealed by sub-5-fs spectroscopy," J. Phys. Chem. B, vol. 105, pp. 413-419, 2001.

[35] H. Kano, T. Saito, and T. Kobayashi, "Observation of herzberg-tellertype wave-packet motion in porphyrin J-aggregates studied by sub-5-fs spectroscopy," J. Phys. Chem. A, vol. 106, pp. 3445-3453, 2002.

[36] A. Colonna, A. Yabushita, I. Iwakura, and T. Kobayashi, "Chirped molecular vibration in a stilbene derivative in solution," Chem. Phys., vol. 341, pp. 336-343, 2007.

[37] T. Kobayashi, T. Saito, and H. Ohtani, "Real-time spectroscopy of transition states in bacteriorhodopsin during retinal isomerization," Nature, vol. 414, pp. 531-534, 2001.

[38] I. Iwakura, A. Yabushita, and T. Kobayashi, "Observation of transition state in Raman triggered oxidation of chloroform in the ground state by real-time vibrational spectroscopy," Chem. Phys. Lett., vol. 457, pp. 421-426, 2008.

[39] A. Anderson, The Raman Effect, Volume 1: Principles. New York: Marcel Dekker, 1971, vol. 1.

[40] T. Takahashi, K. Matsuoka, K. Takimiya, T. Otsubo, and Y. Aso, "Extensive quinoidal oligothiophenes with dicyanomethylene groups at terminal positions as highly amphoteric redox molecules," J. Amer. Chem. Soc., vol. 127, pp. 8928-8929, 2005.

[41] A. Shirakawa, I. Sakane, and T. Kobayashi, "Pulse-front-matched optical parametric amplification for sub-10-fs pulse generation tunable in the visible and near infrared," Opt. Lett., vol. 23, pp. 1292-1294, 1998. 
[42] A. Shirakawa, I. Sakane, M. Takasaka, and T. Kobayashi, "Sub-5-fs visible pulse generation by pulse-front-matched noncollinear optical parametric amplification," Appl. Phys.Lett., vol. 74, pp. 2268-2270, 1999.

[43] A. Baltuska, T. Fuji, and T. Kobayashi, "Visible pulse compression to 4 fs by optical parametric amplification and programmable dispersion control," Opt. Lett., vol. 27, pp. 306-308, 2002.

[44] S. Mukamel, Priciples of Nonlinear Optical Spectroscopy. New York: Oxford Univ. Press, 1995.

[45] R. W. Boyd, Nonlinear Optics. New York: Academic, 1992.

[46] Y. R. Shen, The Principles of Nonlinear Optics. New York: Wiley, 1984.

[47] S. H. Lin, "Rate of interconversion of electronic and vibrational energy," J. Chem. Phys., vol. 44, pp. 3759-3767, 1966.

[48] R. Voltz, "Decay of an excited molecular Born-Oppenheimer state," Mol. Phys., vol. 19, pp. 881-888, 1970.

[49] B. Fluegel, N. Peyghambarian, G. Olbright, M. Lindberg, S. W. Koch, M. Joffre, D. Hulin, A. Migus, and A. Antonetti, "Femtosecond studies of coherent transients in semiconductors," Phys. Rev. Lett., vol. 59, pp. 2588-2591, 1987.

[50] C. H. B. Cruz, J. P. Gordon, P. C. Becker, R. L. Fork, and C. V. Shank, "Dynamics of spectral hole burning," IEEE J. Quantum Electron., vol. 24, no. 2, pp. 261-266, Feb. 1988.

[51] M. Joffre, D. Hulin, J.-P. Foing, J.-P. Chambaret, A. Migus, and A. Antonetti, "Dynamics and Fourier transform studies of the excitonic optical Stark effect," IEEE J. Quantum Electron., vol. 25, no. 12, pp. 2505-2515, Dec. 1989.

[52] J.-P. Likforman, M. Joffre, G. Cheriaux, and D. Hulin, "Control of the spectral-oscillation artifact in femtosecond pump-probe spectroscopy," Opt. Lett., vol. 20, pp. 2006-2009, 1995.

[53] M. Joffre, D. Hulin, A. Migus, A. Antonetti, C. B. a la Guillaume, N. Peyghambarian, M. Lindberg, and S. W. Koch, "Coherent effects in pump-probe spectroscopy of excitons," Opt. Lett., vol. 13, pp. 276-279, 1988.

[54] J.-P. Foing, M. Joffre, J.-L. Oudar, and D. Hulin, "Coherence effects in pump-probe experiments with chirped pump pulses," J Opt. Soc. Amer. B, vol. 10, pp. 1143-1148, 1993.

[55] C. J. Bardeen, Q. Wang, and C. V. Shank, "Femtosecond chirped pulse excitation of vibrational wave packets in LD690 and bacteriorhodopsin," J. Phys. Chem. A, vol. 102, pp. 2759-2766, 1998.

[56] A. T. N. Kumar, F. Rosca, A. Widom, and P. M. Champion, "Investigations of amplitude and phase excitation profiles in femtosecond coherence spectroscopy," J. Chem. Phys., vol. 114, pp. 701-724, 2001.

[57] A. T. N. Kumar, F. Rosca, A. Widom, and P. M. Champion, "Investigations of ultrafast nuclear response induced by resonant and nonresonant laser pulses," J.Chem. Phys., vol. 114, pp. 6795-6815, 2001.

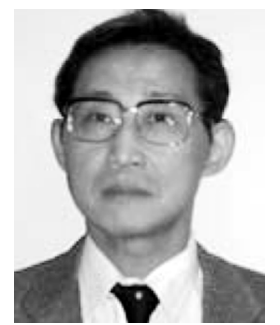

Takayoshi Kobayashi was born in Japan on January 18, 1944. He received the B.S., M. S., and Ph.D. degrees in 1967, 1969, and 1972, respectively, from the University of Tokyo, Tokyo, Japan.

In 1972, he joined the Institute of Physical and Chemical Research (Riken), Saitama Prefecture, Japan, where he was engaged in the picosecond spectroscopy of molecular crystals, molecules in solution, and biological systems. Between 1977 and 1979 he had been a temporary member of the Technical Staff in the Physical and Inorganic Chemistry Department and later in the Coherent Wave Physics Department, Bell Laboratories. At Bell Laboratories, he did picosecond and subpicosecond spectroscopy of organic molecules, metal-organic compounds, charge-transfer complexes, and other systems such as rhodopsins. In 1980, he joined the Department of Physics, University of Tokyo, as a professor. In 2006, he retired from the university and moved to the Department of Applied Physics and Chemistry in the University of Electro- Communications, another National university in Tokyo. At the University of Tokyo, he has been studying picosecond and Femtosecond spectroscopy of polymers, semiconductor doped glasses, and quantum well structured semiconductors, organic molecules, and biological systems. He has been engaged in ultrafast nonlinear optics quantum optics, and chemical physics. At the University of Electro-Communications, he is continuing to be engaged in ultrafast nonlinear optics quantum optics, and chemical physics.

Dr. Kobayashi is a member of the Physical Society of Japan, Japan Society of Applied Physics, Chemical Society of Japan and Spectroscopy Society of Japan. He was awarded the Scientific Achievement Award from the Chemical Society of Japan in 1995, the Scientific Achievement Award from the Society of Spectroscopy of Japan in 2003. He was honored to be a Fellow of the Optical Society of America in 1999. He was awarded the Scientific Achievement Award from the Matsuo Foundation in 2005. He was also awarded the Scientific Achievement Award from the International Vibrational Spectroscopy Conference in 2005.

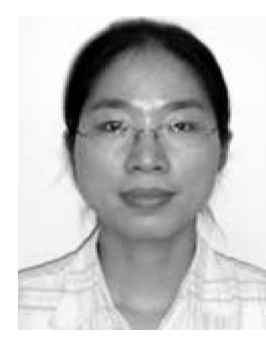

Zhuan Wang was born in Hebei Province, China, on October 28, 1977. She received the B.S. and Ph.D. degrees from Tianjin University in 2000 and 2005, respectively. The research subject for the doctor thesis is the properties of the Kerr lens mode locking Ti:sapphire laser.

In 2005, she joined Takayoshi Kobayashi Group in the University of Tokyo, Japan. The main area of her research is the nonlinear spectroscopy and ultrafast processes in optics and spectroscopy. She is now a Postdoctoral Researcher of Takayoshi Kobayashi Group in the University of Electro-Communications with the research subject of optical physics and ultrafast laser. 